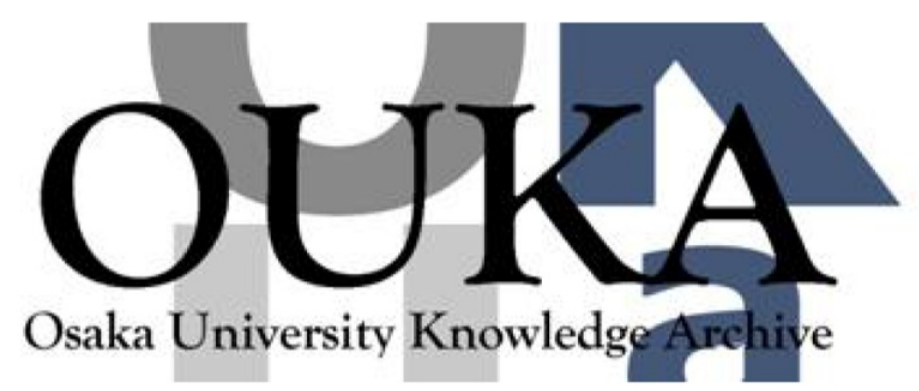

\begin{tabular}{|c|l|}
\hline Title & $\begin{array}{l}\text { Le défini et l' indéfini dans une langue sans } \\
\text { article }\end{array}$ \\
\hline Author(s) & Imoto, Hidetake \\
\hline Citation & Langue française. 171 p. 13-p. 26 \\
\hline Issue Date & $2011-09$ \\
\hline oaire:version VoR \\
\hline URL & https://hdl. handle. net/11094/57760 \\
\hline rights & \\
\hline Note & \\
\hline
\end{tabular}

Osaka University Knowledge Archive : OUKA

https://ir. Library. osaka-u. ac. jp/

Osaka University 


\title{
LE DÉFINI ET L'INDÉFINI DANS UNE LANGUE SANS ARTICLE
} Hidetake Imoto

\author{
Armand Colin | « Langue française »
}

2011/3 n¹71 | pages 13 à 26

ISSN 0023-8368

ISBN 9782200927097

Article disponible en ligne à l'adresse :

http://www.cairn.info/revue-langue-francaise-2011-3-page-13.htm

\section{Pour citer cet article :}

Hidetake Imoto, « Le défini et l'indéfini dans une langue sans article », Langue française 2011/3 ( $\left.\mathrm{n}^{\circ} 171\right)$, p. 13-26.

DOI 10.3917/lf.171.0013

Distribution électronique Cairn.info pour Armand Colin.

(c) Armand Colin. Tous droits réservés pour tous pays.

La reproduction ou représentation de cet article, notamment par photocopie, n'est autorisée que dans les limites des conditions générales d'utilisation du site ou, le cas échéant, des conditions générales de la licence souscrite par votre établissement. Toute autre reproduction ou représentation, en tout ou partie, sous quelque forme et de quelque manière que ce soit, est interdite sauf accord préalable et écrit de l'éditeur, en dehors des cas prévus par la législation en vigueur en France. Il est précisé que son stockage dans une base de données est également interdit. 


\section{Le défini et l'indéfini dans une langue sans article}

\section{INTRODUCTION}

Naoyo Furukawa consacre une grande partie de ses travaux aux différents emplois de l'article et au statut référentiel des syntagmes nominaux en français ${ }^{1}$. Dans la continuité de son travail, j'essaierai, dans cet article, de clarifier le rôle cognitif de la définitude dans l'interprétation d'une phrase, en comparant des syntagmes nominaux français et leurs équivalents japonais. Comme le français est une langue avec articles, les syntagmes nominaux contiennent un déterminant qui marque un trait [ \pm défini]. La définitude est ainsi une propriété inhérente aux déterminants et elle est normalement exprimée. Mais il faut d'abord se demander à quoi sert cette propriété morphosémantique dans une langue. Si elle est indispensable à l'interprétation d'une phrase, pourquoi les langues sans articles ne disposent-elles pas de traits morphologiques qui expriment cette propriété ? De quelle façon s'exprime la différence entre le défini et l'indéfini dans ces langues ? Je tenterais de répondre à ces questions en comparant le français et le japonais.

\section{DÉFINITION}

Tout d'abord, la définitude peut se définir comme trait morphologique indiqué par le déterminant : les articles définis (le, la, les), les adjectifs possessifs et démonstratifs (son, $s a, c e$, etc.) donnent le trait [+défini], les articles indéfinis et partitifs (un, une, des, $d u$, etc.) ainsi que les adjectifs quantitatifs (deux, trois, 
plusieurs, beaucoup de, etc.) donnent le trait [-défini]. En se fondant sur l'hypothèse que ce trait morphologique correspond à une propriété sémantique, beaucoup d'études ont cherché à cerner le caractère essentiel de la définitude sur le plan sémantique. Voici ce qu'elles proposent : présupposition existentielle (Russell 1905), familiarité discursive (Christophersen 1939), unicité (Corblin 1987), accessibilité (Kempson 1988) et identifiabilité (Kempson 1975), etc. Dans la présente étude, je ne traiterai pas directement ce problème sémantique. Je supposerai tout simplement que la définitude est un trait à la fois morphologique et sémantique sans entrer dans les détails. Les noms japonais seront qualifiés comme définis ou indéfinis en fonction de leur traduction en français. Mon hypothèse heuristique est que la dichotomie morpho-sémantique de [ \pm défini] n'est qu'un constituant d'une dichotomie plus globale de la cognition. Cette idée a été inspirée par la notion d'« emballage » (packaging) proposé par W. Chafe (1976) :

[...] a particular noun in a sentence [...] may occupy various "packaging" statuses selected by the speaker on the basis of his assessment of what the addressee's mind is capable of at the time. (Chafe, $1976: 54$ )

Il propose comme tels statuts les six statuts suivants :

(1) a. Le nom peut être ancien ou nouveau (given or new) ;

b. Il peut être un foyer de contraste (focus of contrast) ;

c. Il peut être défini ou indéfini (definite or indefinite);

d. Il peut être le sujet d'une phrase (the subject of the sentence) ;

e. Il peut être le topique d'une phrase (the topic of the sentence) ;

f. Il peut représenter un individu dont le point de vue est adopté par le locuteur ou envers lequel le locuteur éprouve de l'empathie ${ }^{2}$.

Alors qu'il insiste sur la variété de ces statuts, ceux-ci me semblent au contraire très similaires et même interconnectés. Dans un cas prototypique, les statuts ancien, défini, sujet, topique sont réalisés simultanément.

\section{MODĖLE}

Je propose ainsi un modèle dichotomique entre « l'information posée » (abrégée désormais IP) et "l'information à ajouter " (abrégée désormais IA). C'est le modèle le plus abstrait de cognition. L'interprétation d'un énoncé consiste en général à rassembler des informations. Savoir quelque chose, $c^{\prime}$ est associer des IP aux IA. Il me semble que c'est une structure commune au langage qui se concrétise dans chaque langue dans les systèmes dichotomiques suivants :

(2) a. Dichotomie informationnelle information ancienne - - - - information nouvelle [ ancien]

2. It [= the noun] may represent the individual whose point of view the speaker is taking, or with whom the speaker empathizes. (Chafe, $1976: 28$ ) 


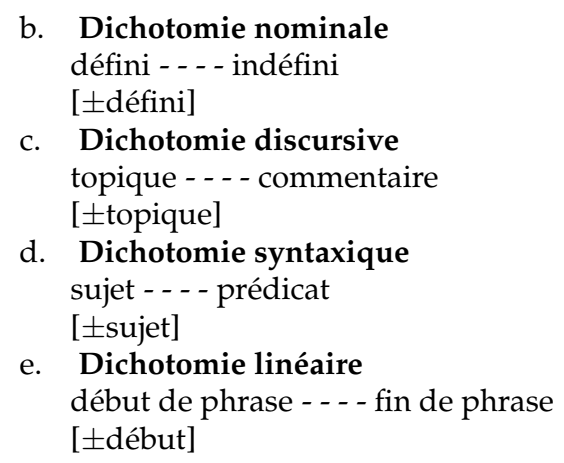

Les traits situés à gauche (ancien, défini, topique, sujet, début) augmentent le degré d'IP et les traits situés à droite (nouveau, indéfini, commentaire, prédicat, fin), celui d'IA. (2) est l'adaptation que je fais des statuts énumérés en (1). (1bf) me semblent être composés de plusieurs facteurs et non structurés par un seul trait objectif, c'est pourquoi ils n'ont pas été inclus dans (2). Je mets pour le moment de côté le trait [+foyer] qui est inextricablement lié aux traits [-ancien] [-défini] [-début]. Mais il est évident que ce trait est un trait positif pour IA.

Les informations anciennes ou nouvelles sont une réalisation directe d'IP et d'IA. Le trait ancien se distingue du trait nouveau par le seul critère de " connu ». Mais il faut considérer l'effet d'accommodation proposé par D. Lewis (1979).

If at time $t$ something is said that requires presupposition $P$ to be acceptable, and if $P$ is not presupposed just before $t$, then -certeris paribus and within certain limitspresupposition $P$ comes into existence at $t$. (Lewis, $1979: 340$ )

La phrase suivante apparaît au début d'un roman :

(3) Jeanne, ayant fini ses malles, s'approcha de la fenêtre, mais la pluie ne cessait pas. [Maupassant, Une vie]

La fenêtre, apparue pour la première fois dans ce roman, est inconnue et en ce sens appartient à l'information nouvelle. Mais le romancier la traitant comme étant déjà connue, la fenêtre en (3) est classée comme information ancienne au niveau de l'interprétation. Il s'agit ici de l'accommodation forcée par l'article défini.

Le trait de la dichotomie nominale [ \pm défini] est attaché morphologiquement à tous les NP français, alors que le japonais qui ne dispose pas d'articles n'a pas de marqueur morphologique obligatoire. Seuls certains adjectifs prénominaux expriment facultativement le défini : kono, sono, ano (démonstratif), et l'indéfini : aru ('certain'), hitotsu no ('un' déterminant numéral cardinal), nanika ('quelque chose'), etc.

La dichotomie discursive distingue le topique du commentaire. Le topique est défini sémantiquement comme 'ce dont on parle' et le commentaire comme 'le 
contenu de ce qu'on dit du topique'. Cette distinction s'applique non seulement à une phrase simple mais à un énoncé connecté.

(4) a. Qu'est-ce qui se passe ?

b. Il y a le facteur qui arrive.

$\mathrm{Si}(4 \mathrm{~b})$ est énoncé à la suite de la question (4a), le topique est ce qui se passe et la phrase toute entière de (4b) devient son commentaire. Le sujet syntaxique est dans beaucoup de cas le topique par défaut.

La dichotomie syntaxique distingue le sujet du prédicat. La définition du sujet par W. Chafe (1976) comme 'ce dont on parle' (what is being talked about) est identique à ma définition du topique. J'aimerais limiter la notion de sujet aux aspects purement syntaxiques et celle de topique aux aspects purement sémantiques. Je définis donc le sujet comme premier argument du verbe principal, le prédicat comme les VP principaux ${ }^{3}$.

La dichotomie linéaire concerne l'ordre linéaire des mots d'une phrase. Le début et la fin dépendent purement de l'ordre linéaire.

\section{INTERACTION}

Chaque dichotomie énumérée en (2) a sa propre logique de distinction, et l'IP que représente la dichotomie morphologique n'est pas exactement la même que celles que représentent les dichotomies informationnelle, discursive, syntaxique ou linéaire. Mais ces systèmes dichotomiques sont plus ou moins dépendants les uns des autres. L'indéfini ne dénote que des informations nouvelles alors que le défini ne dénote pas toujours des informations anciennes ${ }^{4}$.

(5) a. Jean est l'âme damnée de Marie.

b. Jean épousera la femme qui parviendra à escalader ce sommet.

Le topique est inextricablement lié aux informations anciennes et aux définis. D'après K. Lambrecht (1981) :

Topic constituents cannot have referents that are "new" in the discourse; rather their referents must have been mentioned in previous discourse or be salient parts of the situational context of the discourse or be otherwise recoverable. (Lambrecht, $1981: 60$ )

Il indique également que le topique ne peut être indéfini et donne l'exemple suivant :

(6) a. Le garçon, il attend devant la porte.

b. *Un garçon, il attend devant la porte. (Lambrecht, $1981: 61$ )

3. J'adopte ici la structure hiérarchique à deux branches. Il est vrai que la structure syntaxique d'une phrase est souvent compliquée, composée de plusieurs syntagmes (NP, VP, PP, etc.). Mais le premier niveau de séparation est $\mathrm{S} \rightarrow \mathrm{NP}$ (sujet)+VP (prédicat).

4. L'exemple (5) m'a été donné par Anne Zribi-Hertz (communication personnelle). 
T. Givón (1984 : 159) propose le schéma suivant :

$$
\begin{aligned}
& \text { degree of referentiality / topicality } \\
& \text { pronoun }>\text { definite-NP }>\text { indefinite-NP }
\end{aligned}
$$

Le sujet et le défini sont apparemment indépendants, mais on peut remarquer que normalement le sujet est défini. Parmi les 260 phrases du premier chapitre d'Une vie de Maupassant, il y a 196 phrases à sujet défini, 49 phrases à sujet indéfini (y compris 8 on), 15 phrases à impératifs, à interrogatifs ou à vocatifs. Une phrase à sujet indéfini est donc une phrase marquée.

\section{PRINCIPE DES ÉTUDES CONTRASTIVES}

Pour comparer les NP de différentes langues, il est nécessaire de considérer ensemble les traits de l'IP et de l'IA. Le défini, qui est un trait d'IP marqué dans les langues avec article, est exprimé par d'autres traits d'IP dans les langues sans article. Cela a été en effet observé en russe. Selon K. Paykin \& M. van Peteghem (2002 : 98), en russe, langue dépourvue de système d'articles, c'est l'ordre des mots qui permet d'exprimer le défini :

(8) a. Poezd prišël

'train arriva' $\rightarrow$ 'Le train arriva'

b. Prišël poezd 'arriva train' $\rightarrow$ 'Un train arriva'

(8) montre que si le sujet poezd (train) apparaît avant le verbe, il est interprété comme défini, et que s'il apparaît après le verbe, il est interprété comme indéfini. Dans l'exemple suivant, il s'agit de l'ordre entre l'objet direct et l'objet indirect :

(9) a. Maša dala knigu mal'čiku 'Masha donna livre acc $_{\text {garçon }}$ dat $^{\prime} \rightarrow$ 'Masha donna le livre à un garçon'

b. Maša dala mal'čiku knigu

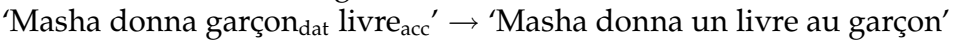
(Paykin \& van Peteghem, 2002 : 98)

En (9) que ce soit un objet direct ou indirect, le défini, en tant qu'IP, apparaît avant l'indéfini. (8) et (9) sont des exemples de l'interprétation de l'opposition défini ou indéfini par l'ordre respectif des NP.

Le trait $[ \pm$ défini] peut être exprimé de manière syntaxique.

(10) a. Ja kupila vody/jablok

'Je achetai eaugen/pommesgen' $\rightarrow$ 'J'ai acheté de l'eau/des pommes'

b. Ja kupila vodu/jabloki

'Je achetai $\mathrm{eau}_{\mathrm{acc}} /$ pommes $_{\mathrm{acc}}{ }^{\prime} \rightarrow$ 'J'ai acheté l'eau/les pommes'

(Paykin \& van Peteghem, 2002 : 100) 
(10) montre qu'un objet génitif est interprété comme indéfini alors qu'un objet accusatif est interprété comme défini. La définitude (la part d'IP) est probablement plus marquée avec l'accusatif qu'avec le génitif. (10) est une réalisation de la dichotomie nominale par une sorte de dichotomie syntaxique.

En japonais, dans la forme parlée, l'objet défini est souvent topicalisé par le mouvement à gauche dans la phrase et par l'absence de postposition. Si l'objet est indéfini, il faut ajouter une marque explicite d'indéfini :

(11) a. Ronbun- $\varphi$ kaita?

'article- $\varphi$ a écrit' $\rightarrow$ 'As-tu écrit l'article ?'

b. Nanika ronbun- $\varphi$ kaita?

'quelque chose article- $\varphi$ a écrit' $\rightarrow$ 'As-tu écrit un article ?'

Ici aussi l'IP du défini en français est transformée en IP du topique et placée au début de la phrase dans l'ordre linéaire en japonais.

\section{COMPARAISON DES NP FRANÇAIS ET DES NP JAPONAIS}

\subsection{Caractéristiques des systèmes dichotomiques IP/IA dans les deux langues}

La dichotomie syntaxique sujet/prédicat est évidente dans les deux langues. En français, le sujet se trouve normalement devant le verbe et et lui impose de s'accorder avec lui ; en japonais, il est marqué par la particule postposée -wa ou $-g a$. Le trait $[ \pm$ sujet] est ainsi donné objectivement dans les deux langues. L'ordre linéaire est également objectif, alors qu'il faut encore fixer le point qui déterminera la limite entre le début et la fin de la phrase. En revanche, ni le français ni le japonais ne possèdent de marque spécifique d'information ancienne/nouvelle. En français, le trait défini est certes normalement attaché à une information ancienne, mais il ne l'est pas toujours comme le montre (5). En japonais, le topique est dans la plupart des cas ancien, mais il y a des exceptions, et le sujet non topique, normalement nouveau, peut être ancien. Le trait [ \pm défini] est toujours marqué morphologiquement par le déterminant en français alors $\mathrm{qu}^{\prime}$ en japonais les marqueurs de ce trait sont facultatifs. En revanche, en japonais, le trait [ \pm topique] est obligatoirement marqué par le choix effectué entre $-w a$ et $-g a$, tandis qu'en français il n'y a qu'une opération marquée de topicalisation comme le mouvement vers la gauche ou l'emploi de l'expression quant à, à propos de, etc. Enfin, la plus grande différence dans la configuration IP/IA consiste dans les dichotomies nominales et discursives ; en français, le système dichotomique du NP est développé au détriment du système discursif alors qu'en japonais, le système dichotomique du discours est développé au détriment de système nominal. 


\subsection{Classification des traits et comparaison}

Passons maintenant à la comparaison concrète. J'aimerais classer les NP suivant leurs traits IP/IA. Comme les traits syntaxiques sont partagés par les deux langues, j'effectuerai un classement en fonction du trait [ \pm sujet].

\subsubsection{Le NP sujet}

Subdivisons le NP sujet suivant le trait [ \pm topique]. Le défini comme l'indéfini apparaissent dans les deux sous-catégories : a. [+sujet], [+topique] ; b. [+sujet], [-topique].

\section{a. [+sujet] [+topique]}

Les deux traits d'IP renforcent le caractère d'IP et amènent aussi les traits [+ancien] [+début] [+défini]. C'est une IP prototypique non marquée dans les deux langues. Les trois marques des traits [+sujet] [+début] [+défini] en français correspondent en japonais aux trois marques des traits [+sujet] [+début] [+topique].

inu-wa chuujitsuna doubutsu da.

chien-TOPIQUE fidèle animal être (présent)

Le chien est un animal fidèle.

Appelons cette correspondance 'correspondance par défaut'.

Le trait [-défini] est marqué dans cette configuration. C'est le cas de ce que l'on appelle « un NP générique » :

(13) a. Le soldat français sait résister à la fatigue.

b. Un soldat français sait résister à la fatigue. (Guillaume, 1973 : 151)

Au niveau du NP, un soldat français, aussi bien que le soldat français, est une IP, et la traduction ne les distingue pas : tous les deux sont traduits en NP topique Furansu-no heishi-wa en japonais. La solution serait au niveau de la phrase : le trait marqué de [-défini] donne une nuance particulière à la phrase toute entière telle qu'un jugement subjectif sur un type idéal. Dans le cas d'une traduction, nous avons recours pour le prédicat à une expression modale. (14ab) correspondent respectivement à (13ab). Ici, le trait [-défini] est exprimé par une expression modale. Appelons ce genre de correspondance 'correspondance particulière avec des traits marqués'.

(14) a. Furansu-no heishi-wa tsukare-ni taeuru.

France-GENITIF soldat-TOPIQUE fatigue-DAT savoir résister

b. Furansu-no heishi-wa tsukare-ni taeuru-monoda.

France-GENITIF soldat-TOPIQUE fatigue-DAT savoir résister-AFFIRMATIF 


\section{b. [+sujet] [-topique]}

En japonais, cette configuration est exprimée par la postposition - $g a$. En français, comme l'indéfini ne peut normalement pas être un topique ${ }^{5}$, la configuration [+sujet] [-topique] [-défini], qui entraîne aussi le trait [-ancien], est presque toujours traduite par une forme $N P-g a$. En plus, si le NP est mis dans la position de foyer ${ }^{6}$ ou après le verbe par l'inversion ([-début]), NP-ga est souvent encore qualifié par un adjectif indéfini marqué.

(15) Mes yeux croisèrent ceux de miss Nelly : il avait montré le bras gauche. Et, ma foi, j'allais en faire nettement la remarque, quand un incident détourna notre attention. [LeBlanc, Arrestation d'Arsène Lupin]

(16) Cependant les cours se font plus étroites, les habitations se rapprochent, les haies disparaissent ; un fagot de fougères se balance sous une fenêtre au bout d'un manche à balai ; il y a la forge d'un maréchal et ensuite un charron avec deux ou trois charrettes neuves, en dehors, qui empiètent sur la route. Puis, à travers une claire-voie, apparaît une maison blanche au delà d'un rond de gazon que décore un Amour, le doigt posé sur la bouche ; deux vases en fonte sont à chaque bout du perron ; des panonceaux brillent à la porte ; c'est la maison du notaire, et la plus belle du pays. [Flaubert, Madame Bovary]

En (15), un incident est traduit par Aru dekigoto-ga (un certain incident), et en (16) une maison blanche est traduit par 1 ken-no Ie-ga (une maison) dont le quantificateur 1 ken-no n'est pas obligatoire sur le plan grammatical.

Enfin, l'indéfini dans la configuration [+sujet] [-topique] [-défini] [-ancien] correspond à la forme NP-ga ([+sujet] [-topique]) par défaut, pourtant l'inverse n'est pas toujours vrai : en français, la configuration [+sujet] [-topique] [+défini] existe aussi. (17a) et sa traduction en français (17b) est un cas typique de cette configuration.

(17) a. What happened? -The dog knocked over the lamp. (Chafe, $1976: 48$ )

b. Qu'est-ce qui se passe ? - Le chien a renversé la lampe.

En (17), la question se comporte comme un topique et la réponse toute entière comme un commentaire. La phrase de réponse représente ce que S.-Y. Kuroda (1973) appelle « jugement thétique », jugement simple, qui s'oppose au " jugement catégorique », jugement double, qui contient l'acte indépendant de jugement, " reconnaissance du sujet ». Nous devons considérer la phrase entière comme IA. Le NP sujet le chien n'est qu'un constituant de l'IA du niveau supérieur. Le trait [+défini] ne contribue donc pas directement à la configuration d'IP/IA. Appelons 'défini indépendant' ce défini qui est attaché à l'un des constituants d'IA et qui n'a pas de relation directe avec la première division d'IP/IA. La lampe comme le chien en (17) sont des définis indépendants. Le choix du trait [+défini] indépendant est dû à la logique propre de la définitude et n'est pas

5. Un NP générique est à part. Voir la section précédente.

6. Il me semble évident qu'il y a une relation étroite entre le foyer et l'IA. 
exprimé par une langue qui ne possède pas de marqueur de ce trait. En effet, en japonais, comme réponse à ce qui se passe, (18ab) sont traduits de manière identique en (18c).

(18) a. Le chien a renversé la lampe.

b. Un chien a renversé la lampe.

c. Inu-ga ranpu-wo taoshita-nda. chien-TOP lampe-ACC a renversé-AFFIRMATIF

(18c) est ambigu hors contexte, mais en principe interprétable de façon univoque en situation d'énonciation. Le défini indépendant indique tout simplement que le référent se trouve dans un domaine préalablement construit (le contexte immédiat, la connaissance de la situation d'énonciation, la connaissance encyclopédique du monde, etc.), tandis que l'indéfini indique que le référent ne se trouve pas dans n'importe quel domaine préalable et qu'il est nouvellement introduit dans le monde raconté. En japonais, il n'y a aucune information sur le domaine dans lequel se trouve le référent. L'interlocuteur le cherche d'abord dans l'un des domaines préalablement construits, et s'il ne l'y trouve pas, il l'interprète comme un élément nouvellement introduit. Dans le cas de (18), si les locuteurs partagent la connaissance qu'il y a un chien et une lampe spécifiques dans la situation d'énonciation, l'interlocuteur identifie ces objets spécifiques comme les référents visés des NP. Ainsi, les traits indépendants qui ne sont pas marqués explicitement sont souvent complétés par les connaissances contextuelles de l'énonciation.

\subsubsection{Le NP non-sujet ([-sujet])}

Normalement une phrase se divise d'abord en deux : le sujet NP et le prédicat $\mathrm{VP}(\mathrm{S} \rightarrow \mathrm{NP}+\mathrm{VP})$. Comme le prédicat tout entier est une IA, le NP avec le trait [-sujet] qui n'est qu'un constituant d'IA est donc indépendant surtout en position de complément d'objet. Pourtant, à l'intérieur d'un PP tel que (19), le défini (la table) se comporte comme IP et s'oppose à l'IA (un livre) :

$$
\text { Il y a un livre sur la table. }
$$

Ce trait [+défini] peut être considéré comme un trait de la configuration plutôt qu'un trait indépendant. Classons le cas de [-sujet] en deux sous-catégories : le NP aux traits indépendants et le NP aux traits de la configuration.

\section{a. Le NP aux traits indépendants}

En position de complément d'objet, les traits attachés à un NP, constituant du VP, sont indépendants. Ils ne sont pas exprimés explicitement en japonais. La distinction interprétative de la valeur [ \pm défini] se fait par les connaissances contextuelles.

(20) a. Le professeur a pris $\{$ le/?un $\}$ train pour aller à la fac.

b. Le professeur a pris $\{u n / ? l e\}$ taxi pour aller à la fac. 
En (20), l'acte de prendre le train ou un taxi étant l'IA de la phrase, le défini le train aussi bien que l'indéfini un taxi est un trait indépendant. Dans notre connaissance du monde, il existe dans les villes un système de transport public normal. $\mathrm{Si}$ cette connaissance encyclopédique est reliée à la connaissance contextuelle d'une ville spécifique, le domaine où on cherche le référent de train est logiquement préconstruit dans notre connaissance contextuelle. Quant à l'indéfini un taxi, on le saisit par l'unité de chaque voiture ; tant qu'il n'y a pas de taxi spécifique préalablement destiné à être pris, on ne cherche pas le référent de taxi dans un domaine préalablement construit, mais on introduit par le NP un taxi un nouveau référent. En ce qui concerne le train et un taxi, il ne s'agit pas d'effectuer un choix entre le défini et l'indéfini. L'article ne reflète que la manière, prédéterminée au niveau lexical, d'actualiser train ou taxi. Le choix de l'article n'ajoute aucune information aux autres constituants de l'énoncé. La simple traduction prendre $\emptyset$ train ou prendre ø taxi suffit à exprimer contextuellement prendre le train ou prendre un taxi.

En position d'attribut dans une phrase copulative, la configuration n'est pas la même suivant que le NP est défini ou indéfini.

(21) a. Paul est un employé d'une entreprise d'eau minérale.

b. Paul est le président d'une entreprise d'eau minérale.

L'IA de la phrase (21a) est le NP un employé d'une entreprise d'eau minérale, et le trait [-défini] est un des traits de la configuration. En revanche, le défini le président en (21b) est un trait indépendant, parce que l'IA de (21b) est le prédicat être le président d'une entreprise d'eau minérale plutôt que le NP le président d'une entreprise d'eau minérale. Dans notre connaissance encyclopédique du terme président, nous savons qu'il n'y a, dans chaque entreprise, qu'un seul président. En conséquence, la connaissance contextuelle d'une entreprise spécifique construit un domaine préalable où on cherche le rôle unique de président d'une entreprise d'eau minérale. C'est l'acte d'assignation de ce rôle à Paul qui est l'IA de la phrase (21b). Ici aussi la simple traduction d'employé et de président suffit à déterminer l'indéfini un employé et le défini le président. Il en est à peu près de même pour (5a) : dans notre connaissance du monde, la locution l'âme damnée est stéréotypée et désigne une seule personne. Le défini l'âme damnée de quelqu'un est lexicalement relié à cette connaissance. Avec cette connaissance lexicale et la connaissance contextuelle attachée à l'individu Marie, le défini l'âme damnée de Marie présuppose le domaine préalable où il existe ce rôle. Cette construction du domaine est faite par l'accommodation que D. Lewis a formulée dans la citation donnée supra (Lewis, 1979 : 340). Ce défini n'entraîne pas le trait [+ancien], mais le caractère préalable qui est essentiel du défini est ainsi assuré.

Dans le cas de (5b), la façon de construire le domaine préalable du référent est un peu différente. Le domaine n'est pas construit par la connaissance encyclopédique du nom femme, mais le contexte immédiat de la phrase relative. Il s'agit ici de deux espaces mentaux : l'un où on aura l'événement décrit dans (22a) et l'autre où l'événement décrit dans (22b) aura lieu. 
(22) a. Une femme parviendra à escalader ce sommet.

b. Jean épousera la femme (en question).

L'espace de (22a) est logiquement préconstruit par rapport à celui de (22b). Même s'il est introduit postérieurement dans l'ordre linéaire, par un effet d'accommodation (cf. citation de Lewis supra), on peut présupposer l'existence du domaine dans lequel le référent de la femme est recherché. Ici encore, la simple traduction de femme qui parviendra à escalader ce sommet suffit à exprimer le caractère défini.

\section{b. Le NP aux traits de configuration}

Une phrase existentielle comme (19) présente la construction presque figée de (23) :

$$
\text { Il y a } \mathrm{NP}_{1} \text { [-défini] préposition } \mathrm{NP}_{2} \text { [+défini] }
$$

qui se compose de la partie IP $\left(\mathrm{NP}_{2}\right)$ et de la partie IA $\left(\mathrm{NP}_{2}\right)$. Sur la scène décrite en (19), la table aussi bien que le livre sont nouvellement introduits dans le discours. Mais en présentant la table comme définie, on la traite comme si elle était préalablement présentée par un effet d'accommodation. Le défini sert ici à placer en arrière-plan l'objet qui est considéré comme l'élément d'un cadre d'interprétation (cadre de la pièce, par exemple). D'ailleurs, le PP dans/sur/devant + NP forme souvent un cadre de lieu. Le NP d'un tel PP est normalement défini. Dans mon corpus de deux millions de mots de la littérature française, la combinaison (24a), c'est-à-dire avec les prépositions dans, sur ou devant et l'article défini, présente 10677 occurrences, alors que la combinaison (24b) ne présente que 2994 occurrences, soit $78 \%$ contre $22 \%$ des occurrences. Dans les phrases qui commencent par il y $a$, si le PP se trouve jusqu'à 7 mots après $i l y a$, on a le résultat de 43 occurrences de (25a) (82,7\%) contre 9 occurrences de (25b) (17,3\%).

(24) a. $\quad\{$ dans/sur/devant $\}$ \{le/la/l' $\}$

b. $\{$ dans/sur/devant $\}$ un/une $\}$

(25) a. Il y a... (deux à sept mots quels qu'ils soient)... \{dans/sur/devant $\}\{$ le/la/l' $\}$

b. Il y a... (deux à sept mots quels qu'ils soient)... \{dans/sur/devant $\}\{$ un/une $\}$

Dans les occurrences d'il y a avec un PP contenant un NP indéfini de mon corpus littéraire, le NP est non spécifique comme en (26) :

- Croirais-tu ? Marius rentre à présent à des une heure du matin! Bahorel répondait : - Que veux-tu ? il y a toujours un pétard dans un séminariste. [Hugo, Les Misérables]

Le seul exemple attesté d'un PP contenant un NP indéfini spécifique est le suivant :

(27) Sur la photo, on ne voit d'abord qu'un énorme prisme de granit fendu, le glacier hérissé de pointes et un éboulis enneigé. Mais l'un des blocs est surmonté par une tête coiffée d'un bonnet mongol. Un homme est assis 
là, enveloppé dans un manteau sombre. Devant lui, il y a une toile sur un chevalet. [Le Monde, 23 octobre 2003]

En (27), le PP devant lui est IP et la phrase entière il y a une toile sur un chevalet est IA. Ainsi, la phrase il y a un livre sur la table (19) est plus canonique que (28) ; en ce sens qu'en (28) les deux NP indéfinis ne constituent pas une dichotomie informationnelle :

(28) Il y a un livre sur une table.

L'acte d'accommodation exécuté souvent sur le PP défini de la phrase existentielle est une opération pour rendre l'énoncé dichotomique.

L'équivalent japonais de l'exemple (19) est le suivant (29) :

(29) teeburu-no ue-ni hon-ga aru. table-GEN haut-LOC libre-NOM exister

Cette phrase s'interprète difficilement comme équivalent à (28) bien que le NP teeburu n'ait aucune marque de défini. L'interprétation définie de teeburu vient principalement du sens même de la phrase existentielle. En japonais aussi, une phrase existentielle combine un objet d'existence (IA) et un lieu où il se trouve (IP). L'interprétation définie est encore étayée par l'ordre de la phrase. En (29), le syntagme postpositionnel se trouvant au début de la phrase (cet ordre me semble plus canonique que hon-ga teeburu no ue-ni aru, qui est aussi grammaticalement correct $^{7}$ ) amène à penser que cette table est un objet déjà connu. Les traits [+début] [+défini] [+ancien] collaborent ainsi dans l'interprétation d'IP. Enfin, on peut remarquer la correspondance par défaut entre le PP défini de la phrase existentielle française et $\mathrm{N}-n i$ de la phrase existentielle japonaise.

Pour exprimer le PP indéfini en (27) et en (28), le japonais doit avoir recours à une expression marquée : on ajoute un marqueur explicite d'indéfini toaru (certain) devant teeburu.

(30) a. Toaru teburu-no ue-ni hon-ga aru. certaine table GEN haut-LOC livre-NOM exister 'Sur une certaine table, un livre existe.'

b. Hon-ga toaru teburu-no ue-ni aru. livre-NOM certaine table-GEN haut-LOC exister 'Un livre existe sur une certaine table.'

Dans ce cas-là, il n'y a pas de préférence dans l'ordre linéaire entre table et livre. Même s'il est le seul trait de la configuration $\mathrm{d}^{\prime} \mathrm{IP}^{8}$, le trait [+défini] est le trait par défaut du PP de la phrase existentielle. Le trait [-défini] est, par conséquent, un trait marqué pour le NP d'un syntagme prépositionnel de lieu. Il s'agit ici de la correspondance particulière par un trait marqué.

7. Même dans ce cas-là, on interprète par défaut teeburu comme défini, à cause du sens de la phrase.

8. En (19), les autres traits de la table sont [-topique] [-sujet] [-début] ([-ancien] au moins contextuellement). 
Il en est à peu près de même pour l'exemple suivant :

La voiture, arrêtée un instant, reprit donc sa marche, traversa la foule, continua son chemin, enfila la rue Saint-Honoré, tourna la rue des BonsEnfants et s'arrêta devant une porte base. [Dumas, Les trois mousquetaires]

Bien qu'il se trouve à l'intérieur du prédicat, avec les traits [-défini] [-ancien] [-début] et encore avec le trait [+foyer], le NP une porte basse se comporte comme IA au premier niveau. En effet, on est amené à diviser la phrase en deux avec la voiture s'arrêta (IP) d'un côté et devant une porte basse (IA) de l'autre ; l'acte de s'arrêter étant information attachée à la voiture. Le trait [-défini] est ainsi, jusqu'à un certain degré, un trait de la configuration. Dans la traduction publiée des Trois mousquetaires, une porte basse de (31) est traduit en aru ookina mon, de la même façon que pour la traduction d'une table en (30). C'est un autre exemple de la correspondance par un trait marqué : comme en (28), le trait [-défini] est marqué à l'intérieur d'un PP.

\section{CONCLUSION}

Le NP comporte tout un ensemble de traits dichotomiques explicites ou implicites suivant lesquels le NP s'interprète comme information posée (IP) ou comme information à ajouter (IA) dans un énoncé. La définitude est l'un des traits d'IP qui sont marqués explicitement en français, mais qui ne sont exprimés qu'implicitement en japonais. En considérant les autres traits d'un NP qui sont inextricablement liés à la définitude et en faisant une comparaison avec l'équivalent japonais, on arrive à cerner le rôle et la caractéristique de ce trait. Dans le cas où le défini est un trait de la configuration, c'est-à-dire un trait qui contribue directement à l'interprétation d'IP, le trait [+défini] est exprimé en tant qu'IP par les autres traits d'IP en japonais. C'est le cas de ce que j'ai appelé correspondance par défaut où le trait [+topique], le trait [-topique], le trait [+début] correspondent respectivement au défini, à l'indéfini ainsi qu'à la structure même du PP dans une phrase existentielle. Si le trait [-défini] est un trait marqué, c'està-dire utilisé au lieu de [+défini] qui est par défaut, en japonais aussi on utilise une expression spéciale. C'est le cas de ce que j'ai appelé 'correspondance par des traits marqués' où l'expression modale et l'ajout de l'adjectif indéfini facultatif $a r u$ correspondent respectivement à l'indéfini du sujet d'un NP générique et à l'indéfini du NP d'un PP. Dans le cas où le défini est un trait indépendant, c'est-àdire un trait d'un NP qui se trouve à l'intérieur d'une IA et qui ne contribue pas directement à la configuration d'IP/IA, il n'est pas du tout exprimé en japonais. L'équivalent japonais du NP en question n'est cependant pas ambigu sur le plan de la définitude, car l'information donnée par le trait [ \pm défini] indépendant est complétée par la connaissance contextuelle. Considérer tous les traits d'un NP en même temps ouvre ainsi la possibilité de mettre au clair le rôle de chaque trait et de fournir un critère de comparaison des NP à travers différentes langues. 


\section{Références bibliographiques}

CHAFE W. (1976), “Givenness, contrastiveness, definiteness, subjects, topic, and point of view", Subject and topic, 27-55.

ChRISTOPHERSEN P. (1939), The Articles: A study of their theory and use in English, Copenhagen: Einar Munksgaard.

CoRbuin F. (1987), Indéfini, défini et démonstratif. Construction linguistique de la référence, Paris/Genève : Droz.

FuRUKAWA N. (1986), L'article et le problème de la référence en français, Tokyo : France Tosho.

FurukaWA N. (1997), "Les Glaneuses de Millet: emploi intensionnel de LE(S) ", Revue de Sémantique et Pragmatique 2, 169-181.

Furukawa N. (1998), "Cet objet curieux qu'on appelle l'article : emploi de l'article défini dans des environnements métalinguistiques ", in A. Englebert et al. (éds), La ligne claire. Mélanges offerts à Marc Wilmet à l'occasion de son $60^{e}$ anniversaire, Louvain-la-Neuve : Duculot, 47-54.

FurukaWa N. (2000), "À propos de l'emploi 'quasi-intentionnel' de l'article défini ; la copie du dessin et a copy of the drawing ", in A. Englebert et al. (éds), Actes du XXIle congrès international de linguistique et de philologie romanes. Sens et signification, Tübingen : Niemeyer, 243-250.

Furukawa N. (2009), "Article défini et métaphore : étude descriptive ", L'information grammaticale 123, 10-16.

FurukaWA N. (2010), "L'article défini et le problème dit de l'unicité : quantité ou qualité ? ", Bulletin d'Études de Linguistique Française 44, 65-82.

GIvón T. (1984), Syntax: a functional-typological introduction, Amsterdam: John Benjamins.

Guillaume G. (1973), Langage et science du langage, Paris : Nizet.

KEMPSON R. (1975), Presupposition and the delimitation of semantics, Cambridge: Cambridge University Press.

KEMPSON R. (1988), “Grammar and conversational principles", in F. Newmeyer (ed.), Linguistics: the Cambridge Survey, vol. II: Linguistic theory: extensions and implications, Cambridge : Cambridge University Press, 139-163.

KURODA S.-Y. (1973), "Le jugement catégorique et le jugement thétique ; exemples liés de la syntaxe japonaise ", Language 30, 81-110.

LAMBRECHT K. (1981), Topic, Antitopic and Verb-agreement in Non-Standard French, Amsterdam: John Benjamins.

LEWIS D. (1979), “Scorekeeping in a Language Game”, Journal of Philosophical Logic 8, 339-359.

PAykin K. \& VAN Peteghem M. (2002), “Definiteness in a language without articles", Recherches linguistiques de Vincennes 31, 97-112.

Russell B. (1905), “On denoting”, Mind 14, 479-493.

ZRIBI-HERTZ A. (2002), "The DP hypothesis and the syntax of identification”, Recherches linguistiques de Vincennes 31, 127-142. 